\title{
Proper motion of a nuclear jet relative to a maser feature in NGC 3079
}

\author{
Satoko Sawada-Satoh \\ ISAS, Yoshinodai, Sagamihara, Kanagawa, 229-8510 JAPAN \\ Makoto Inoue, Katsunori M. Shibata, Seiji Kameno \\ NAO, Mitaka, Tokyo, 181-8588 JAPAN \\ Naomasa Nakai \\ NRO, Nobeyama, Minamimaki, Nagano, 384-1305 JAPAN \\ Victor Migenes \\ Univ. de Guanajuato, Guanajuato, CP 36000 MEXICO \\ Phil J. Diamond \\ JBO, Jodrell Bank, Macclesfield, Cheshire, SK11 9DL U.K.
}

\begin{abstract}
We present high resolution studies of the nuclear region in NGC 3079 with phase-referenced VLBI monitoring between its water maser and continuum emission. From results of the observations, NGC 3079 showed relative motion between core-jet-like continuum components. One of the components (A) relative to the reference maser feature moved with an apparent subluminal velocity along the SE-NW direction, almost parallel to alignment of the components. On the other hand, the position of the other component (B) did not show such a significant change. If the maser gas is associated with a rotating disk, the maser gas should be gravitationally bound to the central mass. This suggests that $\mathrm{B}$ and $\mathrm{A}$ are the nucleus and jet, respectively.
\end{abstract}

\section{Introduction}

High resolution images of megamaser sources allow us to gain vital insights into the nuclear regions of galaxies. A few known megamaser sources have been imaged with VLBI, which has provided glimpses of the parsec-scale region in AGN. Miyoshi et al. (1995) conducted high resolution observation of NGC 4258 with VLBI and found that an edge-on thin Keplerian disk surrounding a $3 \times$ $10^{7} \mathrm{M}_{\odot}$ black hole. Such views are not available with any other methods.

In NGC 4258, the dynamical center of the disk, or location of the nucleus, was determined (Herrnstein et al. 1997). The exact location of the nucleus in 
an AGN provides us a reference position for multi-frequency and multi-epoch observations. This location is useful for AGN studies, astrometry and so on.

NGC 3079 is a water megamaser source with peak flux of $\sim 3 \mathrm{Jy}$. The main maser features of the emission cover the velocity range of $930-1070 \mathrm{~km} \mathrm{~s}^{-1}$ in $V_{\text {LSR }}$, blue-shifted from the systemic velocity of the galaxy (Henkel et al. 1984, Haschick \& Baan 1985). Two faint features with velocities around $1190 \mathrm{~km} \mathrm{~s}^{-1}$ (Nakai et al. 1995) and at $1123 \mathrm{~km} \mathrm{~s}^{-1}$ (Trotter et al. 1998; hereafter T98) are also found.

It also has core-jet-like radio continuum components: two stronger components (A and B) and one fainter component $(\mathrm{C})$ between them, along the SE-NW direction in the nuclear region (Irwin \& Seaquist 1988; hereafter IS88). VLBI observations by T98 reveal that water maser emission arises in compact clumps and is distributed over $\sim 2$ pc along the N-S direction, which has no positional coincidence with the continuum components. Two 5- GHz continuum

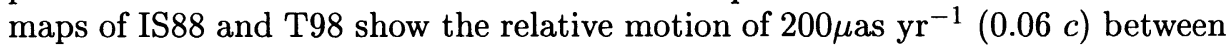
component $\mathrm{A}$ and $\mathrm{B}$.

\section{Observations}

We conducted multi-epoch VLBI observations of the nuclear region in NGC 3079, over 13 months from October 1996 to October 1997. Thirteen stations were used; the VLBA, the VLA, the Green Bank 43-m telescope and the Effelsberg 100-m telescope. In order to detect weak continuum emission, a phase-referencing technique was applied using the brightest maser feature as a reference. Continuum channels were produced by averaging those channels containing no spectral line emission after residual delay, rate, phase and amplitude corrections.

\section{Results and Discussions}

By the use of the phase-referencing technique, we see the relative positions of maser features and continuum components at $22 \mathrm{GHz}$ (Fig. 1). The three epoch maser distributions and the spatial separation between the maser groups and the continuum components from October 1996 to October 1997 are consistent with each other, and also agree well with the map of T98 in January 1995. No significant motion of the maser groups are seen during the 32 months from January of 1995 (T98) to October of 1997.

However, the monitoring observations at $22 \mathrm{GHz}$ show changes in the relative position of the continuum components from the reference maser feature (Fig. 2). The position of A relative to the reference maser feature moved with an apparent subluminal velocity of $0.13 \pm 0.04 c$ from January of 1995 to October of 1997, along the SE-NW direction (P.A. of $132 \pm 12^{\circ}$ ). The apparent velocity and the direction of the motion of $\mathrm{A}$ relative to the reference maser feature are similar to those of the subluminal motion between A and B. On the other hands, the position of $B$ did not show such a rapid change. This suggests that all maser features are gravitationally bound to the central mass, which is B, or is located very close to $B$ rather than $A$.

Radio continuum spectra of the components also support the hypothesis that $A$ and $B$ are the jet and nucleus, respectively. Simultaneous multi-frequency 

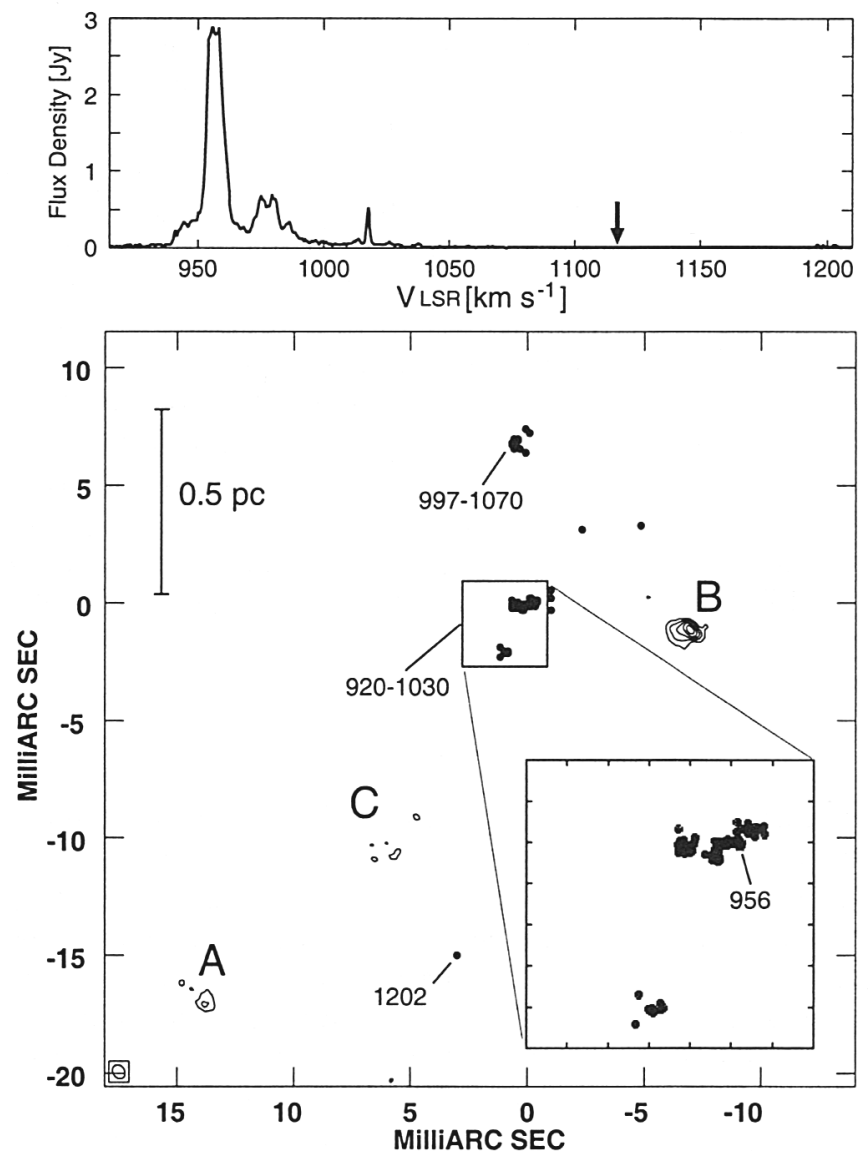

Figure 1. (top) Cross-power spectra of the maser emission of NGC 3079 measured on 1997 March. The arrow indicates the systemic velocity of the galaxy. (bottom) Distribution of maser features overlayed on the continuum contours in the nuclear region. The brightest maser at $956 \mathrm{~km} \mathrm{~s}^{-1}$ was used as a reference.

VLBI observation shows steep spectrum of A and convex spectrum of B between frequencies at 1.4 and $22 \mathrm{GHz}$ (Sawada-Satoh et al. 2000). A nuclear system with the convex spectrum exhibited by $\mathrm{B}$ and the steep spectrum associated with A is similar to the well known core-jet morphology.

\section{Conclusions}

We have indentified component $B$ as the nucleus and omponent $A$ as a jet feature in the nuclear region of NGC 3079, by measuring thier proper motions with respect to the masers. Continuum spectra of the components also support our identifications. Maser features in AGNs can provide us a stable reference position in the nuclear region. 

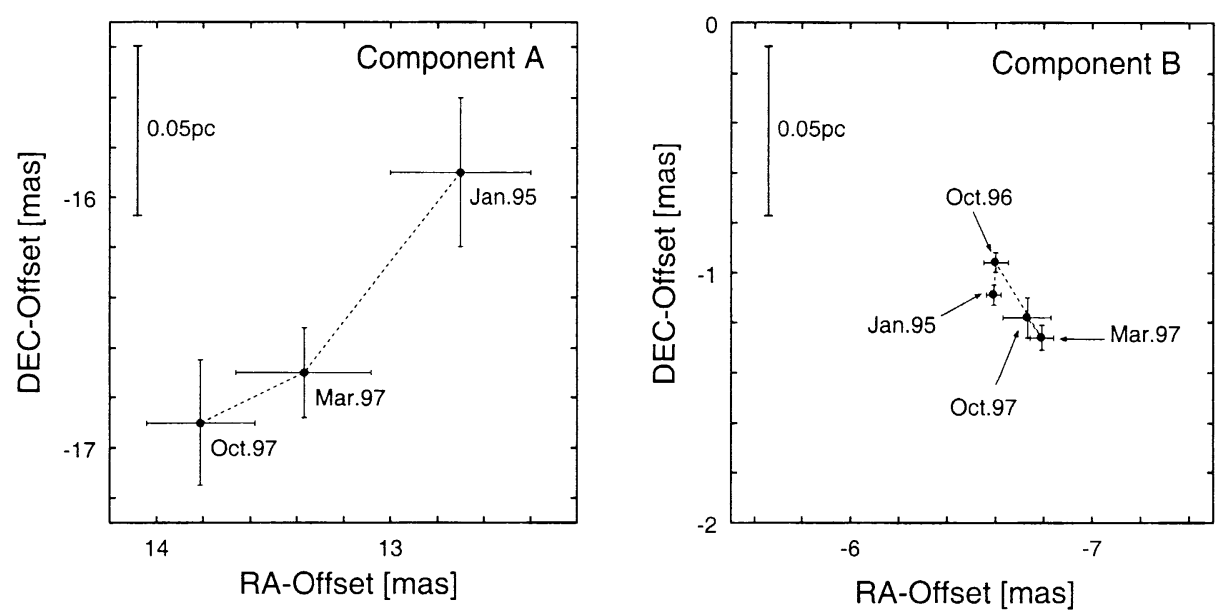

Figure 2. Motion of the components A and B from the reference maser feature at $956 \mathrm{~km} \mathrm{~s}^{-1}$. We obtained the single positions by fitting a single Gaussian model of the images. The positions for January 1995 were estimated by $\mathrm{T} 98$.

\section{Acknowledgement}

The VLBA is operated by the National Radio Astronomy Observatory, which is a facility of the National Science Foundation operated under cooperative agreement by Associated Universities, Inc.

\section{References}

Haschick, A. D. \& Baan, W. A. 1985, Nature, 314, 144.

Henkel, C., Güsten, R., Downes, D., Thum, C., Wilson, T. L. \& Biermann, P. 1984, A\&A, 141, L1.

Herrnstein, J. R., Moran, J. M., Greenhill, L. L., Diamond, P. J., Miyoshi, M., Nakai, N. \& Inoue, M. 1997, ApJ, 475, L17.

Irwin, J. A. \& Seaquist, E. B. 1988, ApJ, 335, 658 (IS88)

Miyoshi, M., Moran, J. M., Herrnstein, J. R., Greenhill, L. J., Nakai, N., Diamond, P. J. \& Inoue, M. 1995, Nature, 373, 771.

Nakai, N., Inoue, M., Miyazawa, K., Miyoshi, M. \& Hall, P. 1995, PASJ, 47, 771.

Sawada-Satoh, S., Inoue, M., Shibata, K. M., Kameno, S., Migenes, V., Nakai N.\& Diamond, P. J. 2000, PASJ, 52, 421.

Trotter, A. S., Greenhill L. J., Moran, J. M., Reid, M. J., Irwin, J. A. \& Lo, K. Y. 1998, ApJ, 495, 740. (T98) 